\title{
Lesões condrais maiores tratadas com uso de membrana de colágeno - condrogênese autóloga induzida por matriz - apresentam maior aumento nos escores clínicos*
}

\section{Larger Chondral Lesions Treated with Collagen Membrane - Matrix-Induced Autologous Chondrogenesis - Show Larger Increase in Clinical Scores}

\author{
Mateus Kenji Christo Miyahira1(1) João Victor Novaretti ${ }^{2(0)}$ Diego Costa Astur ${ }^{2(1)}$ \\ Camila Cohen Kaleka ${ }^{1}$ Joicemar Tarouco Amaro ${ }^{1}$ Moisés Cohen ${ }^{2}$ \\ Paulo, SP, Brasil \\ Endereço para correspondência Mateus Kenji Christo Miyahira, \\ Instituto Cohen, Av Lineu de Paula Machado, 660, Cidade Jardim, São \\ Paulo, SP, 05601-000, Brasil (e-mail: kenjimiyahira23@hotmail.com).
}

\footnotetext{
${ }^{1}$ Instuto Cohen de Ortopedia, Reabilitação e Medicina do Esporte, São

2 Departamento de Ortopedia e Traumatologia, Escola Paulista de Medicina, Universidade Federal de São Paulo, São Paulo, SP, Brasil
}

Rev Bras Ortop 2021;56(3):333-339.

\begin{abstract}
Resumo
Objetivo Avaliar clínica e radiologicamente os resultados do tratamento das lesões condrais com a membrana de colágeno - condrogênese autóloga induzida por matriz. Métodos Trata-se de uma série de casos observacional, na qual foram analisados 15 pacientes submetidos a condrogênese autóloga induzida por matriz. A avaliação clínica foi feita comparando os escores de Lysholm e International Knee Document Commitee (IKDC, na sigla em inglês) no pré- e pós-operatório de 12 meses, e avaliação radiológica através do escore de Magnetic Resonance Observation of Cartilage Repair Tissue (MOCART, na sigla em inglês) no mesmo período de pós-operatório.

Resultados A média de idade dos pacientes foi 39,2 anos, e a média do tamanho das

Palavras-chave

- cartilagem articular

- condrogênese

- colágeno

- traumatismos do joelho lesões condrais foi de $1,55 \mathrm{~cm}^{2}$. Houve uma melhora significativa nos escores clínicos, com média de aumento de 24,6 pontos no Lysholm e de 24,3 no IKDC, após 12 meses. $\mathrm{Na}$ avaliação radiológica, o MOCART teve média de 65 pontos. Observou-se que quanto maior o tamanho da lesão, maior foi a melhora nos escores.

Conclusão Avaliando escores clínicos subjetivos, o tratamento das lesões condrais com a membrana de colágeno mostrou bons resultados, assim como a avaliação de
\end{abstract}

Trabalho desenvolvido no Departamento de Ortopedia e Traumatologia, Universidade Federal de São Paulo (UNIFESP), São Paulo, SP, Brasil. recebido

11 de Dezembro de 2019

aceito

02 de Março de 2020

Publicado on-line

Setembro 25, 2020
DOI https://doi.org/ $10.1055 / \mathrm{s}-0040-1712493$. ISSN 0102-3616. (c) 2020. Sociedade Brasileira de Ortopedia e Traumatologia. All rights reserved.

This is an open access article published by Thieme under the terms of the Creative Commons Attribution-NonDerivative-NonCommercial-License, permitting copying and reproduction so long as the original work is given appropriate credit. Contents may not be used for commercial purposes, or adapted, remixed, transformed or built upon. (https://creativecommons.org/ licenses/by-nc-nd/4.0/)

Thieme Revinter Publicações Ltda., Rua do Matoso 170, Rio de Janeiro, RJ, CEP 20270-135, Brazil 
MOCART, com maior benefício em lesões maiores.

\begin{abstract}
Keywords

- articular cartilage

- chondrogenesis

- collagen

- knee injuries

Objective To evaluate clinically and radiologically the results of the treatment of chondral lesions using collagen membrane - autologous matrix-induced chondrogenesis (AMIC). Methods This is a series of observational cases, in which 15 patients undergoing AMIC were analyzed. The clinical evaluation was made by comparing the Lysholm and International Knee Document Commitee (IKDC) scores in the pre- and postoperative period of 12 months, and radiological evaluation using the Magnetic Resonance Observation of Cartilage Repair Tissue (MOCART) score in the same postoperative period.

Results The mean age of the patients was 39.2 years old, and the mean size of the chondral lesions was $1.55 \mathrm{~cm}^{2}$. There was a significant improvement in clinical scores, with a mean increase of 24.6 points on Lysholm and of 24.3 on IKDC after 12 months. In the radiological evaluation, MOCART had a mean of 65 points. It was observed that the larger the size of the lesion, the greater the improvement in scores.

Conclusion Evaluating subjective clinical scores, the treatment of chondral lesions with the collagen membrane showed good results, as well as the evaluation of MOCART, with greater benefit in larger lesions.
\end{abstract}

\section{Introdução}

A função biomecânica da matriz de proteoglicanos e fibras colágenas da cartilagem é absorver cargas compressivas e tensionais que atuam sobre a articulação. ${ }^{1}$ Lesões da cartilagem são observadas em até $11 \%$ das artroscopias, sendo metade dessas $>2 \mathrm{~cm}^{2}{ }^{2}$ A maioria tem relação com trauma ou osteocondrite dissecante.

A capacidade de cura da cartilagem é limitada ${ }^{3,4}$ podendo levar a osteoartrose. ${ }^{5} \mathrm{Um}$ dos grandes desafios para o cirurgião ortopédico continua a ser o tratamento das lesões condrais. ${ }^{6}$ Devido ao baixo potencial de cura, e ao grau de desconforto que essas lesões causam, a intervenção cirúrgica tem sido amplamente usada na tentativa de preencher os defeitos cartilaginosos. ${ }^{7,8}$ As células mesenquimais são a fonte para regeneração. ${ }^{9}$

Pridie, ${ }^{10}$ em 1959, foi o primeiro a realizar estímulo ao reparo utilizando sangue oriundo da medula óssea. Steadman et al., ${ }^{11}$ em 2001, introduziram a técnica das microfraturas. A microfratura é a técnica para recrutar as células mesenquimais, pois o coágulo promove um meio favorável para o tecido de reparo cartilaginoso. ${ }^{12} \mathrm{O}$ tecido cicatricial "fibrocartilagem-like"13 previne a osteoartrose e melhora os sintomas dos pacientes. ${ }^{14}$

Como o coágulo formado não possui boa resistência mecânica, foi proposto o uso da membrana de colágeno. ${ }^{15-17}$ A condrogênese autóloga induzida por matriz (AMIC, na sigla em inglês) é uma técnica que combina as microfraturas com a membrana de colágeno. ${ }^{18}$ Enquanto as microperfurações estão indicadas para defeitos $<2 \mathrm{~cm}^{2}$, a AMIC é indicada para defeitos maiores. ${ }^{19}$

O objetivo do presente estudo é avaliar clinica e radiologicamente os resultados do tratamento das lesões condrais do joelho com uso da membrana de colágeno - AMIC.

\section{Metodologia}

O presente estudo é uma série de casos observacional de pacientes com lesões condrais do joelho, operados entre 2015 e 2018 com utilização de membrana de colágeno pela técnica AMIC e aprovado pelo comitê de ética da instituição.

Todos os pacientes participantes do presente estudo foram esclarecidos sobre a técnica cirúrgica indicada e outras opções de tratamento, suas vantagens e desvantagens, e concordaram com a realização do procedimento escolhido.

As indicações foram pacientes entre 15 e 55 anos de idade, com lesões condrais de 0,5 a 5,0 centímetros de diâmetro, na patela, tróclea ou côndilos femorais, graus III ou IV de ICRS (Internacional cartilage repair society), ativos e sintomáticos. Não foram incluídos pacientes com história de cirurgia prévia para cartilagem, mau-alinhamento do membro inferior acometido ou instabilidade ligamentar não corrigidos previamente ou concomitantemente ao procedimento. Os critérios de exclusão foram pacientes que abandonaram o acompanhamento ambulatorial ou não concordaram em participar.

Um total de 15 pacientes foram incluídos no estudo, 12 homens e 3 mulheres, todos operados pelo mesmo cirurgião.

\section{Avaliação pré-operatória}

Os pacientes que apresentaram suspeita de lesão condral no joelho, de acordo com anamnese e exame físico, realizaram exames de imagem complementares para conclusão diagnóstica, com radiografias e ressonância magnética (RM), podendo caracterizar e medir a lesão condral e identificar lesões ligamentares ou mau alinhamento dos membros inferiores. Dessa forma, foram identificados os 


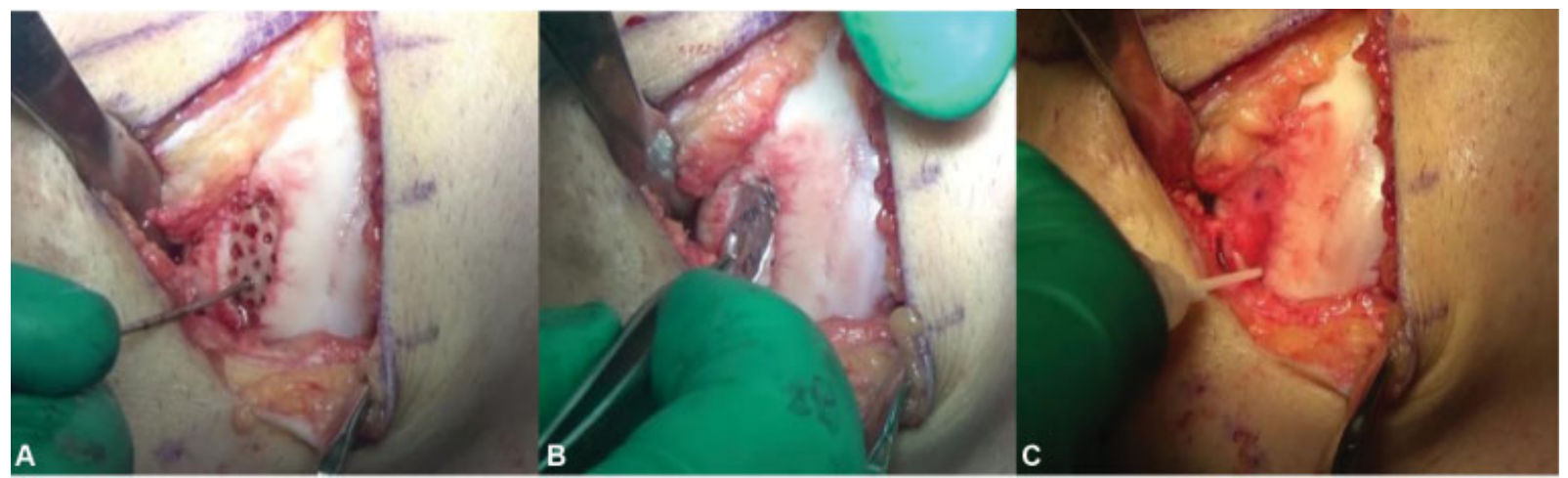

Fig. 1 A, área de lesão condral desbridada e microperfurada. B, molde metálico usado para medir dimensões da lesão. C, aspecto final mostrando a membrana de colágeno fixada pela cola de fibrina.

pacientes que preenchiam os critérios para inclusão no estudo.

\section{Técnica cirúrgica}

Primeiramente, era realizada artroscopia do joelho acometido, para localizar a lesão condral, e avaliar os ligamentos e meniscos. Então, posteriormente, uma artrotomia parapatelar longitudinal era realizada de acordo com a região articular a ser operada. Quando houve algum outro procedimento cirúrgico concomitante, como reconstrução do ligamento cruzado anterior ou osteotomia valgizante da tíbia, a condroplastia foi realizada sempre por último.

Após desbridamento do defeito condral, retirada de tecido cartilaginoso degenerado e inviável, era realizada a curetagem do leito da lesão retirando toda camada calcificada e preservando o osso subcondral (- Figura $\mathbf{1}$ ).

Utilizando um template metálico, era definido com precisão o tamanho e formato da lesão, a membrana de colágeno tipo I/III de origem porcina (Chondrogide; Geistlich Pharma AG, Wolhusen, Suíça) que iria cobrir o defeito era recortada. Em seguida, eram realizadas microperfurações no osso subcondral, à mão livre com $2 \mathrm{a} 4 \mathrm{~mm}$ de distância entre eles. A membrana era então colocada sobre o defeito e fixada provisoriamente com duas agulhas. A fixação definitiva da membrana era feita com fio absorvível monocryl 5.0 e complementada com cola de fibrina nas bordas da lesão.

\section{Reabilitação}

Apesar dos diferentes procedimentos cirúrgicos associados ao uso da membrana de colágeno, o protocolo de reabilitação se iniciou com 3 semanas de imobilização com brace articular e sem descarga de peso no membro operado.

Com o objetivo de reduzir o quadro inflamatório, até a $5^{\text {a }}$ semana era iniciado o aumento da amplitude de movimento e ativação do músculo quadríceps. Após isso, até a $8^{\mathrm{a}}$ semana, o paciente foi incentivado a deambular sem uso de órtese, progressivamente, até normalizar a marcha. Entre 6 e 8 meses, o paciente era liberado para praticar esportes de contato.

\section{Coleta de dados}

Dados demográficos como idade, sexo, lateralidade, tamanho e localização da lesão condral, e procedimentos asso- ciados foram coletados do banco de dados. A avaliação clínica pré-operatória foi feita com os escores de Lysholm ${ }^{20}$ e International Knee Document Commitee (IKDC), ${ }^{21}$ e a pósoperatória com um ano de seguimento, através do Lysholm, IKDC, e o escore radiológico de Magnetic Resonance Observation of Cartilage Repair Tissue (MOCART). ${ }^{22}$ Este escore é um sistema de pontuação que busca avaliar o tecido de reparo em sua extensão, intensidade do sinal, preenchimento do defeito, integração com a cartilagem adjacente, entre outros.

\section{Análise estatística}

Inicialmente, todas as variáveis foram analisadas descritivamente. Para as variáveis quantitativas, esta análise foi feita através da observação dos valores mínimos e máximos, e do cálculo de médias, desvios padrão (DPs) e quartis. Para as variáveis qualitativas, calcularam-se frequências absolutas e relativas. Para a comparação de médias de dois momentos de avaliação, foi utilizado o teste $t$ de Student pareado. ${ }^{23}$ Para o estudo das correlações entre os deltas dos escores e variáveis avaliadas no estudo, foi utilizado o coeficiente de correlação de Pearson. O software utilizado para os cálculos foi o SPSS Statistics for Windows, Version 17.0 (SPSS Inc., Chicago, IL, EUA). O nível de significância utilizado para os testes foi de $5 \%$.

\section{Resultados}

Foram avaliados 15 pacientes com idade entre 15 e 54 anos (média de 39,2 anos), sendo 3 mulheres e 12 homens. As lesões acometeram a tróclea femoral em seis casos, a patela em cinco casos e os côndilos femorais em quatro casos. $\mathrm{Na}$ - Tabela 1, apresenta-se a distribuição de frequências do local da lesão.

O tamanho das lesões variou de $0,6 \mathrm{~cm}^{2}$ até $2,34 \mathrm{~cm}^{2}$, medidos através das RMs pré-operatórias, e o índice de massa corporal (IMC) variou de $21,6 \mathrm{~kg} / \mathrm{m}^{2}$ até $32,5 \mathrm{~kg} / \mathrm{m}^{2}$, conforme mostra a $\mathbf{- T a b e l a} \mathbf{2}$ com os valores descritivos dessas variáveis.

Os escores Lysholm e IKDC foram avaliados nos momentos pré- e pós-cirurgia, com 12 meses. Houve aumento significativo nas médias do escore de Lysholm (55,9 versus 80.5$)$ e 
Tabela 1 Distribuição de frequências do local da lesão para os 15 pacientes avaliados

\begin{tabular}{|l|l|l|}
\hline Local da lesão & $\mathbf{n}$ & $\%$ \\
\hline CFM + CFL & 1 & 6,7 \\
\hline CFM D & 3 & 20,0 \\
\hline Patela D & 3 & 20,0 \\
\hline Patela E & 2 & 13,3 \\
\hline Tróclea D & 4 & 26,7 \\
\hline Tróclea E & 2 & 13,3 \\
\hline Total & 15 & 100,0 \\
\hline
\end{tabular}

Abreviações: CFL, côndilo femoral lateral; CFM, côndilo femoral medial; d, direita; e, esquerda.

Tabela 2 Valores descritivos do tamanho da lesão e do IMC dos 15 pacientes

\begin{tabular}{|l|l|l|l|l|l|}
\hline Variável & $\mathbf{n}$ & Média & $\mathbf{d p}$ & Mínimo & Máximo \\
\hline Tamanho & 15 & 1,55 & 0,58 & 0,60 & 2,34 \\
\hline IMC & 15 & 27,6 & 2,6 & 21,6 & 32,5 \\
\hline
\end{tabular}

Abreviações: dp, desvio padrão; IMC, índice de massa corporal.

do escore IKDC (51,6 versus 75,9) do pré- para o pósoperátorio $(\mathrm{p}<0.001)$ ( - Figuras 2 e 3 ).

Os escores Tegner e Mocart foram avaliados em um único momento e estão descritos na - Tabela 3. Através dos resultados obtidos com as RMs após 1 ano, foi mostrado que todos os pacientes mantiveram o preenchimento da lesão condral com tecido de reparo, com boa integração das bordas. A média do escore de MOCART foi de 65 pontos, variando de 50 a 75.

Para o estudo de possíveis correlações entre variáveis e a variação pré e pós-operatória dos escores, foi calculado o delta de variação dos escores apresentados na - Tabela 4.

$\mathrm{Na}$ - Tabela 5 apresentam-se os coeficientes de correlação entre a idade, tamanho da lesão e IMC e os deltas de variação dos escores Lysholm e IKDC. Há correlação positiva e significativa entre o tamanho da lesão e o delta de variação do escore IKDC. Portanto, quanto maior o valor do tamanho da lesão, maior o delta de variação do escore IKDC (-Figura 4). Não foi encontrada relação entre a melhora observada e a idade e IMC.

\section{Discussão}

O achado mais importante do presente estudo foi que pacientes com lesões condrais de maior tamanho tiveram um maior aumento nos escores clínicos, portanto um maior benefício com o tratamento. Esses resultados mostram concordância com o estudo anterior publicado pelo grupo. ${ }^{24} \mathrm{Não}$ houve impacto significante da idade e IMC nos resultados encontrados neste estudo.

A técnica AMIC vem sendo utilizada por diversos cirurgiões, sendo estabelecida como uma opção no tratamento dos defeitos cartilaginosos. Já foi mostrada em estudos anteriores a capacidade das microfraturas no osso subcondral em levar células mesenquimais da medula óssea para a região da cartilagem, promovendo o aporte de fatores de crescimento e citocinas. Testes in vitro já demonstraram que a membrana de colágeno pode reter as células mesenquimais, podendo melhorar a capacidade regenerativa da técnica de microfraturas. ${ }^{15}$

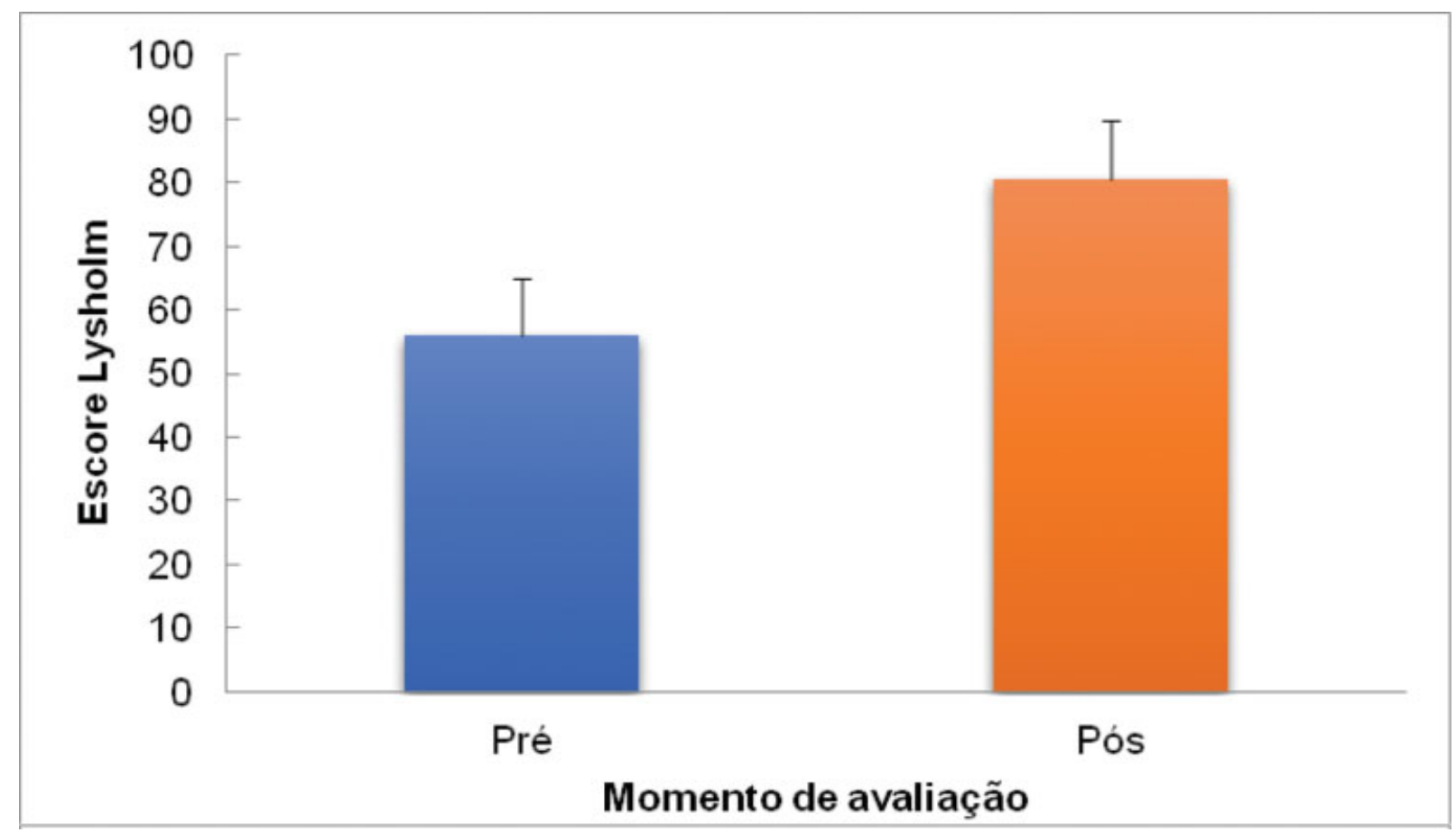

Fig. 2 Média e desvio-padrão do escore Lysholm no pré- e pós-operatório. 


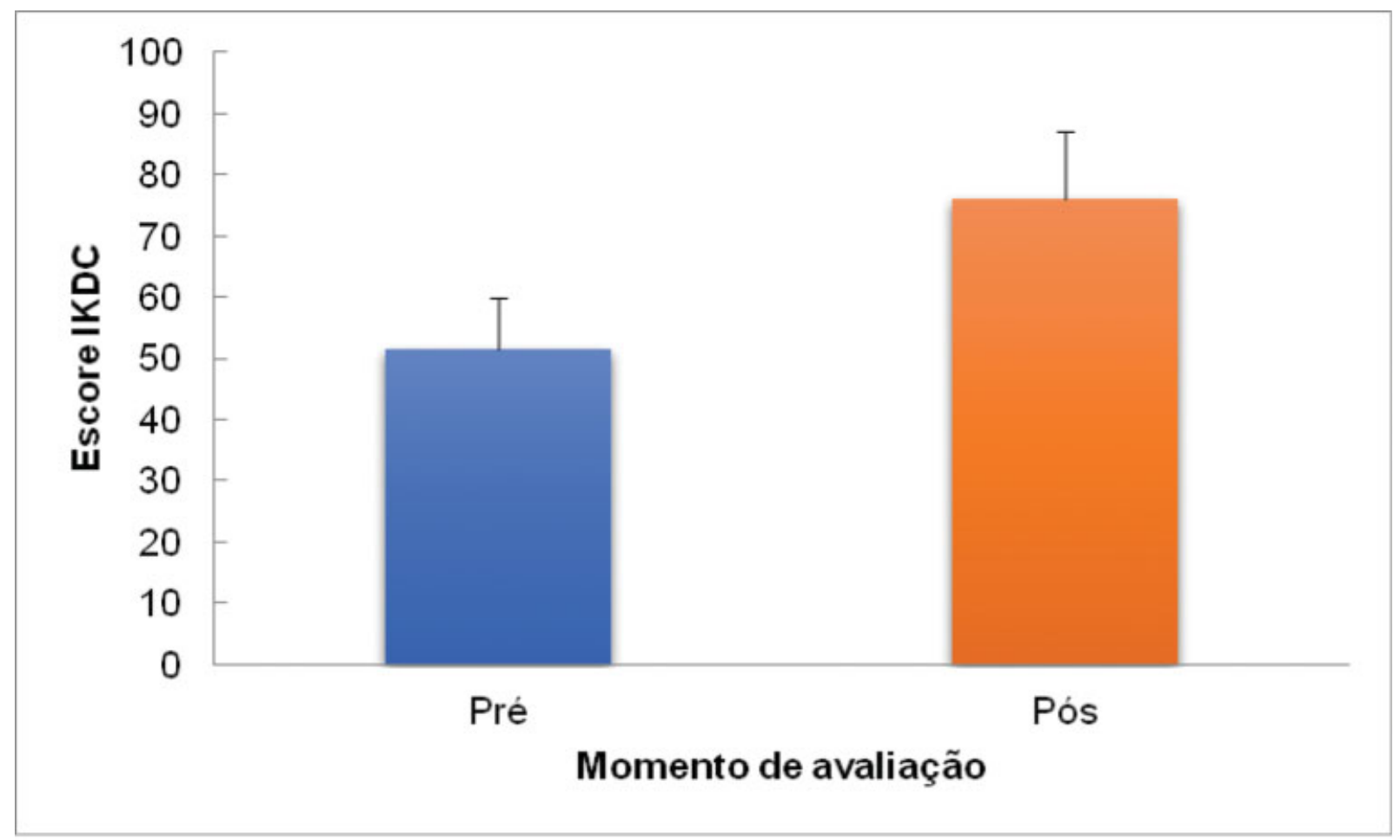

Fig. 3 Média e desvio-padrão do escore International Knee Documentation Committee (IKDC, na sigla em inglês) no pré- e pós-operatório.

Tabela 3 Valores descritivos dos escores Tegner e Mocart dos 15 pacientes

\begin{tabular}{|l|l|l|l|l|l|}
\hline Variável & $\mathbf{n}$ & Média & $\mathbf{d p}$ & Mínimo & Máximo \\
\hline Tegner & 15 & 3,9 & 1,1 & 2,0 & 6,0 \\
\hline Mocart & 15 & 65,0 & 7,8 & 50,0 & 75,0 \\
\hline
\end{tabular}

Abreviação: dp, desvio padrão.

Tabela 4 Valores descritivos dos escores deltas de variação dos escores Lysholm e IKDC dos 15 pacientes

\begin{tabular}{|l|l|l|l|l|l|}
\hline Variável & $\mathbf{n}$ & Média & $\mathbf{d p}$ & Mínimo & Máximo \\
\hline Lysholm & 15 & 24,6 & 9,0 & 11,0 & 41,0 \\
\hline IKDC & 15 & 24,3 & 7,1 & 12,6 & 33,4 \\
\hline
\end{tabular}

Abreviação: dp, desvio padrão.

Um estudo de 2008 comparou os resultados do tratamento das lesões condrais com microfraturas, utilizando ou não a membrana de colágeno em lesões acetabulares. Foi mostrado que a uma melhora mais sustentada dos escores clínicos foi alcançada com a utilização da membrana. ${ }^{25}$

Comparado com outros procedimentos cirúrgicos para tratamento dos defeitos cartilaginosos, a combinação das microfraturas com a membrana de colágeno é uma boa opção, com apenas um tempo cirúrgico, de baixo custo, sem morbidade de área doadora sadia ou necessidade de proliferação celular in vitro, como no transplante osteocondral autólogo e o transplante autólogo de condrócitos, res-
Tabela 5 Coeficientes de correlação de Pearson

\begin{tabular}{|l|l|l|l|}
\hline \multirow{4}{*}{ Idade } & & Delta Lysholm & Delta IKDC \\
\hline \multirow{4}{*}{ Tamanho } & $\mathrm{r}$ & 0,266 & $-0,188$ \\
\cline { 2 - 4 } & valor-p & 0,338 & 0,502 \\
\cline { 2 - 4 } & $\mathrm{n}$ & 15 & 15 \\
\cline { 2 - 4 } & $\mathrm{r}$ & $-0,143$ & 0,593 \\
\cline { 2 - 4 } & valor-p & 0,610 & 0,020 \\
\cline { 2 - 4 } & $\mathrm{n}$ & 15 & 15 \\
\hline \multirow{5}{*}{ IMC } & $\mathrm{r}$ & 0,450 & $-0,017$ \\
\cline { 2 - 4 } & valor-p & 0,092 & 0,953 \\
\cline { 2 - 4 } & $\mathrm{n}$ & 15 & 15 \\
\hline
\end{tabular}

Abreviação: IMC, índice de massa corporal.

pectivamente. A mesma técnica do presente estudo, realizada artroscopicamente, já foi descrita por Piontek et al., apresentando resultados promissores. ${ }^{26}$ Schagemann et al., ${ }^{27}$ em 2018, compararam a técnica artroscópica do AMIC com a técnica mini open, semelhante à do presente trabalho, concluindo não haver diferença nos resultados a médio prazo, com 2 anos de seguimento.

Dhollander et al. ${ }^{28}$ já reportaram melhoras satisfatórias após 2 anos de pós-operatório, porém uma tendência à deterioração do tecido de reparo, analisado pela RM. Nesta série de casos, as melhoras foram boas ou excelentes. Através dos exames de imagem, não foram observados afilamento ou hipercrescimento dos tecidos de reparo. Outros estudos já demonstraram não haver melhora clínica no mesmo ritmo da evolução do 


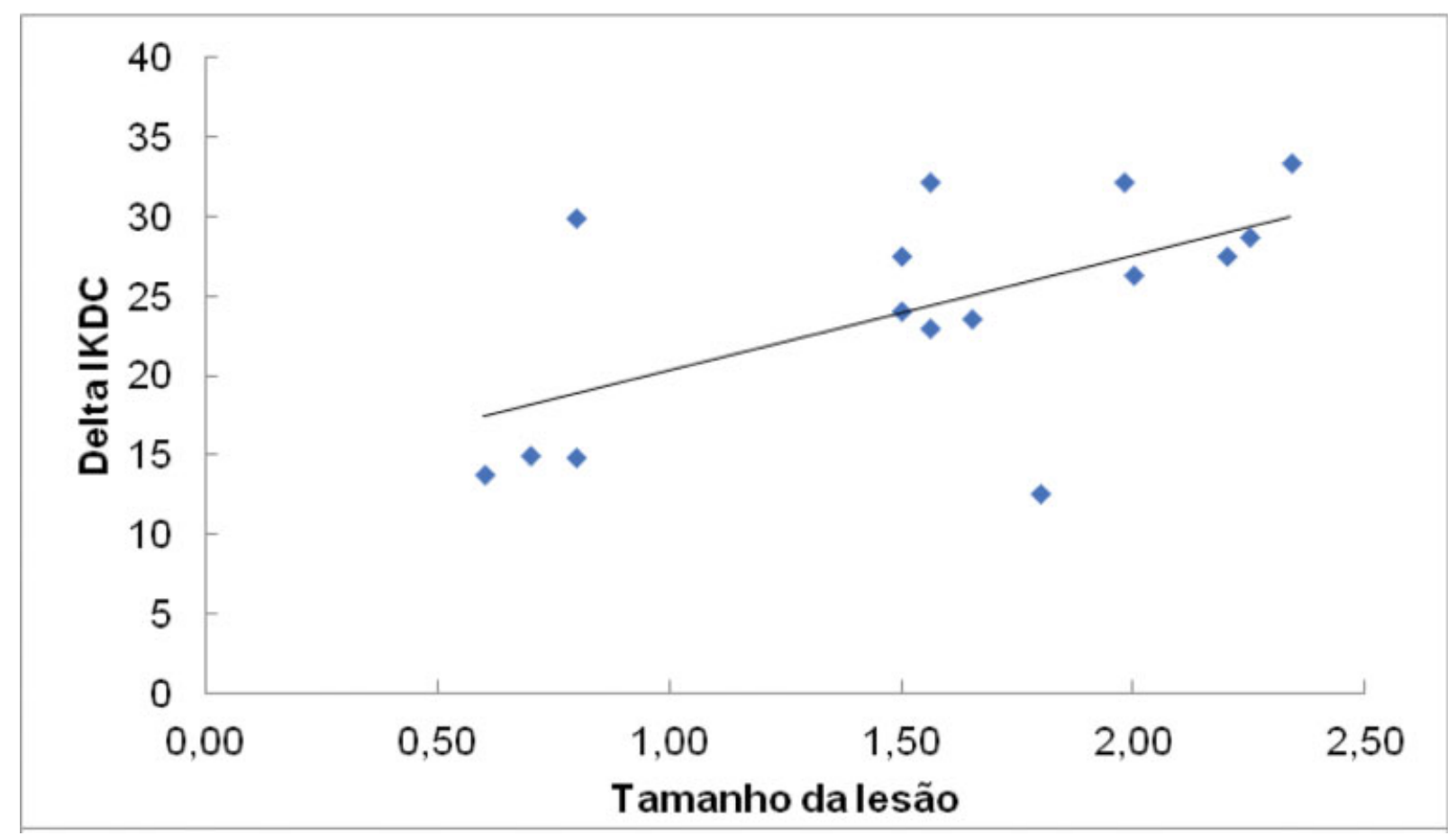

Fig. 4 Gráfico de dispersão do tamanho da lesão e o delta de variação do escore International Knee Documentation Committee (IKDC, na sigla em inglês.).

tecido de reparo observado pelas RMs. ${ }^{22}$ Acreditamos que a avaliação radiológica possa ser um indicativo de falha do tratamento, porém não um indicativo de sucesso.

Existem limitações que devem ser consideradas ao avaliar o presente trabalho. $O$ pequeno número de pacientes reflete uma realidade para os cirurgiões do país, pelo acesso ao material necessário ao procedimento. Devido a isso, as lesões são descritas em partes diferentes da articulação, com variados procedimentos concomitantes, tornando a amostra mais heterogênea.

\section{Conclusão}

Avaliando escores clínicos subjetivos, o tratamento das lesões condrais com a membrana de colágeno mostrou bons resultados, assim como a avaliação de MOCART, e que existe maior benefício em lesões maiores.

\section{Suporte Financeiro}

Não houve suporte financeiro de fontes públicas, comerciais, ou sem fins lucrativos.

Conflito de Interesses

Os autores declaram não haver conflito de interesses.

\section{Referências}

1 Gottardi R, Hansen U, Raiteri R, et al. Supramolecular Organization of Collagen Fibrils in Healthy and Osteoarthritic Human Knee and Hip Joint Cartilage. PLoS One 2016;11(10):e0163552

2 Arøen A, Løken S, Heir S, et al. Articular cartilage lesions in 993 consecutive knee arthroscopies. Am J Sports Med 2004;32(01): 211-215
3 Buckwalter JA, Mankin HJ. Articular cartilage: degeneration and osteoarthritis, repair, regeneration, and transplantation. Instr Course Lect 1998;47:487-504

4 Raimondi MT, Bonacina E, Candiani G, et al. Comparative chondrogenesis of human cells in a 3D integrated experimentalcomputational mechanobiology model. Biomech Model Mechanobiol 2011;10(02):259-268

5 Heir S, Nerhus TK, Røtterud JH, et al. Focal cartilage defects in the knee impair quality of life as much as severe osteoarthritis: a comparison of knee injury and osteoarthritis outcome score in 4 patient categories scheduled for knee surgery. Am J Sports Med 2010;38(02):231-237

6 Farr J, Gomoll AH. 2016 barriers to cartilage restoration. J Clin Orthop Trauma 2016;7(03):183-186

7 Kubosch EJ, Erdle B, Izadpanah K, et al. Clinical outcome and T2 assessment following autologous matrix-induced chondrogenesis in osteochondral lesions of the talus. Int Orthop 2016;40(01):65-71

8 Steinwachs M. New technique for cell-seeded collagen-matrixsupported autologous chondrocyte transplantation. Arthroscopy 2009;25(02):208-211

9 Kon E, Filardo G, Di Matteo B, Perdisa F, Marcacci M. Matrix assisted autologous chondrocyte transplantation for cartilage treatment: A systematic review. Bone Joint Res 2013;2(02): $18-25$

10 Pridie K. A method of resurfacing osteoarthritic knee joints. J Bone Joint Surg Br 1959;41:618-619

11 Steadman JR, Rodkey WG, Rodrigo JJ. Microfracture: surgical technique and rehabilitation to treat chondral defects. Clin Orthop Relat Res 2001;(391, Suppl)S362-S369

12 Richter W. Mesenchymal stem cells and cartilage in situ regeneration. J Intern Med 2009;266(04):390-405

13 Hunziker EB. Articular cartilage repair: basic science and clinical progress. A review of the current status and prospects. Osteoarthritis Cartilage 2002;10(06):432-463

14 Kusano T, Jakob RP, Gautier E, Magnussen RA, Hoogewoud H, Jacobi M. Treatment of isolated chondral and osteochondral defects in the knee by autologous matrix-induced chondrogenesis 
(AMIC). Knee Surg Sports Traumatol Arthrosc 2012;20(10): 2109-2115

15 Bark S, Piontek T, Behrens P, Mkalaluh S, Varoga D, Gille J. Enhanced microfracture techniques in cartilage knee surgery: Fact or fiction? World J Orthop 2014;5(04):444-449

16 Gille J, Behrens P, Volpi P, et al. Outcome of Autologous Matrix Induced Chondrogenesis (AMIC) in cartilage knee surgery: data of the AMIC Registry. Arch Orthop Trauma Surg 2013;133(01):87-93

17 Benthien JP, Behrens P. Autologous matrix-induced chondrogenesis (AMIC). A one-step procedure for retropatellar articular resurfacing. Acta Orthop Belg 2010;76(02):260-263

18 Behrens P. Matrixgekoppelte Mikrofrakturierung. Arthroskopie 2005; $18: 193-197$

19 Anders S, Martin Volz, Frick H, Gellissen J. A randomized,controlled trial comparing autologus matrix inducedchondrogenesis (AMIC) to microfracture: analysis of 1- and2-year follow-up data of 2 centers. Open Orthop J 2013;7(Suppl 1):M4:133-143

20 Peccin MS, Ciconneli R, Cohen M. Questionário específico para sintomas do joelho Lysholm Knee Scoring Scale -Tradução e validação para a língua Portuguesa. Acta Ortop Bras 2006;14(05):268-272

21 Hefti F, Müller W, Jakob RP, Stäubli HU. Evaluation of knee ligament injuries with the IKDC form. Knee Surg Sports Traumatol Arthrosc 1993;1(3-4):226-234

22 Marlovits S, Striessnig G, Resinger CT, et al. Definition of pertinent parameters for the evaluation of articular cartilage repair tissue with high-resolution magnetic resonance imaging. Eur J Radiol 2004;52(03):310-319

23 Rosner B. Fundamentals of Biostatistics. $2^{\text {nd }}$. Boston: PWS Publishers; 1986

24 Astur DC, Lopes JC, Santos MA, Kaleka CC, Amaro JT, Cohen M. Surgical treatment of chondral knee defects using a collagen membrane - autologus matrix-induced chondrogenesis. Rev Bras Ortop 2018;53(06):733-739

25 Fontana A, de Girolamo L. Sustained five-year benefit of autologous matrix-induced chondrogenesis for femoral acetabular impingement-induced chondral lesions compared with microfracture treatment. Bone Joint J 2015;97-B(05): 628-635

26 Piontek T, Ciemniewska-Gorzela K, Szulc A, Naczk J, Słomczykowski M. All-arthroscopic AMIC procedure for repair of cartilage defects of the knee. Knee Surg Sports Traumatol Arthrosc 2012;20 (05):922-925

27 Schagemann J, Behrens P, Paech A, et al. Mid-term outcome of arthroscopic AMIC for the treatment of articular cartilage defects in the knee joint is equivalent to mini-open procedures. Arch Orthop Trauma Surg 2018;138(06):819-825

28 Dhollander A, Moens K, Van der Maas J, Verdonk P, Almqvist KF, Victor J. Treatment of patellofemoral cartilage defects in the knee by autologous matrix-induced chondrogenesis (AMIC. Acta Orthop Belg 2014;80(02):251-259 